\title{
A semisynthetic diterpenoid lactone inhibits NF-кB signalling to ameliorate inflammation and airway hyperresponsiveness in a mouse asthma model
}

\begin{abstract}
Andrographolide (AGP) and 14-deoxy-11,12-didehydroandrographolide (DDAG), two main diterpenoid constituents of Andrographis paniculata were previously shown to ameliorate asthmatic symptoms in a mouse model. However, due to inadequacies of both compounds in terms of drug-likeness, DDAG analogues were semisynthesised for assessment of their antiasthma activity. A selected analogue, 3,19-diacetyl-14-deoxy-11,12didehydroandrographolide (SRS27), was tested for inhibitory activity of NF- $\kappa$ B activation in TNF- $\alpha$-induced A549 cells and was subsequently evaluated in a mouse model of ovalbumin (OVA)-induced asthma. Female BALB/c mice, 6-8 weeks old were sensitized on days 0 and 14, and challenged on days 22, 23 and 24 with OVA. Compound or vehicle (3\% dimethyl sulfoxide) was administered intraperitoneally $1 \mathrm{~h}$ before and $11 \mathrm{~h}$ after each OVA aerosol challenge. On day 25, pulmonary eosinophilia, airway hyperresponsiveness, mucus hypersecretion, inflammatory cytokines such as IL-4, -5 and -13 in BAL fluid, gene expression of inflammatory mediators such as 5-LOX, E-selectin, VCAM-1, CCL5, TNF- $\alpha$, AMCase, Ym2, YKL-40, Muc5ac, CCL2 and iNOS in animal lung tissues, and serum IgE were determined. SRS27 at $30 \mu \mathrm{M}$ was found to suppress NF- $\kappa \mathrm{B}$ nuclear translocation in A549 cells. In the ovalbumin-induced mouse asthma model, SRS27 at $3 \mathrm{mg} / \mathrm{kg}$ displayed a substantial decrease in pulmonary eosinophilia, BAL fluid inflammatory cytokines level, serum IgE production, mucus hypersecretion and gene expression of inflammatory mediators in lung tissues. SRS27 is the first known DDAG analogue effective in ameliorating inflammation and airway hyperresponsiveness in the ovalbumin-induced mouse asthma model.
\end{abstract}

Keyword: NF-кB; A549 cell line; Andrographolide analogues; Ovalbumin; Oxidative stress; Airway hyperresponsiveness; Mouse asthma model 
